STRUCTURAL BIOLOGY COMMUNICATIONS

ISSN 2053-230X

Keywords: crystallization; ICCBM15

\section{Introduction to selected articles from the 15th ICCBM}

\author{
Howard Einspahr, ${ }^{\text {a* }}$ Ivana Kuta Smatanova, ${ }^{\mathrm{b}}$ Christian Betzel $^{\mathrm{c}}$ and Jeroen Mesters ${ }^{\mathrm{d}}$ \\ aPO Box 6483, Lawrenceville, NJ 08648-0483, USA, ' University of South Bohemia Ceske Budejovice, Faculty of \\ Science, Institute of Chemistry and Biochemistry \& Academy of Sciences of the Czech Republic, Branisovska 1760, \\ CZ-370 05 Ceske Budejovice, Czech Republic, 'Laboratory for Structural Biology of Infection and Inflammation, \\ Department of Chemistry, c/o DESY, University of Hamburg, Notkestrasse 85, 22603 Hamburg, Germany, and Institute \\ of Biochemistry, Center for Structural and Cell Biology in Medicine, University of Lübeck, Ratzeburger Allee 160, 23538 \\ Lübeck, Germany. *Correspondence e-mail: hmeinspahr@yahoo.com
}

The July 2015 issue of Acta Crystallographica Section F, Structural Biology Communications features a special selection of papers that elaborate on presentations made at the 15th International Conference on the Crystallization of Biological Macromolecules (ICCBM15), which was held in Hamburg, Germany, 17-20 September 2014.

The first international meeting of this kind was held in 1985 at Stanford, California. ICCBM15, organized under the umbrella of the International Organization for Biological Crystallization (IOBCr), represents an unbroken span of 30 years of these biennial meetings. With 300 conference participants, the continuing and growing interest of crystallographers, chemists, physicists and engineers in crystal growth is confirmed, and this special section is a fine example of the range of research that these meetings continue to attract and support.

The articles are a useful addition to Acta Cryst. $F$ and cover important structural biology topics related to crystallization. These include progress in nucleation studies, new techniques for detection of crystalline precipitates, production and scoring of nano- and micro-sized crystal suspensions in support of emerging applications of X-ray free-electron laser data collection procedures. These and more are examples of responses to the continued challenges to produce crystals for structural biology, featured at the Hamburg meeting.

We draw your attention to this special section of the issue, confident you will find much of interest you might not encounter elsewhere in your reading, and we hope that it whets your appetite for ICCBM16 which will be held on 2-7 July 2016 in Prague, Czech Republic. 